Bol. Acad. peru. leng. 63. 2018 (155-183)

\title{
ANÁLISIS FONÉTICO-FONOLÓGICO DE LOS PROCESOS QUE AFECTAN A LOS SEGMENTOS OCLUSIVOS EN POSICIÓN DE CODA EN EL CASTELLANO LIMEÑO
}

\section{PHONETIC-PHONOLOGICAL ANALYSIS OF THE PROCESSES AFFECTING THE OCCLUSIVE SEGMENTS IN THE CODA POSITION IN THE SPANISH OF LIMA}

\author{
Óscar Esaul Cueva Sánchez \\ Universidad Nacional Mayor de San Marcos
}

\section{Resumen:}

El presente trabajo se propone dar una descripción sincrónica de los procesos fonológicos que sufren los segmentos oclusivos en posición de coda al interior de palabra en el castellano limeño; además, la parte descriptiva de la investigación se apoyará en datos acústicos que a lo largo del trabajo podrán evidenciarse; para este propósito se usó el programa Praat (análisis acústico). Por otro lado, el contraste entre el arranque y la coda silábica se evidencia a partir de los tipos de procesos que afectan a cada una de estas posiciones; por ejemplo, los arranques tienden a modificarse por fenómenos de reforzamiento, mientras que las codas silábicas se modifican por procesos de debilitamiento. Justamente, este trabajo se interesa por dar cuenta de estos procesos de debilitamiento que afectan a los segmentos oclusivos en coda silábica; asimismo, para la parte explicativa del trabajo se usará como marco teórico a la fonología generativa no lineal. 


\section{Abstract:}

This paper is intended to give a synchronic description of the phonological processes suffered by the occlusive segments in coda position at the inner part of the word in the Spanish of Lima. In addition, the descriptive part of the research will be supported by acoustic data that may be evidenced throughout the work. For this purpose the Praat program was used (acoustic analysis). On the other hand, the contrast between the onset and syllabic coda is evidenced from the types of processes that affect each of these positions; for example, the onsets tend to be modified by reinforcement phenomena, while the syllabic codas are modified by weakening processes. Precisely, the purpose of this work is to give account of these weakening processes affecting the occlusive segments in syllabic coda. Likewise, for the explanatory part of this paper, the non-linear generative phonology will be used as a theoretical framework.

Palabras clave: Procesos de debilitamiento; coda silábica; fonología generativa.

Key words: Weakening processes; syllabic coda; generative phonology.

Fecha de recepción:

Fecha de aceptación:
$14 / 03 / 2018$

$31 / 05 / 2018$

\section{Introducción}

Es recurrente encontrar en el estudio fonológico de las lenguas un centralismo dedicado a estudiar los elementos que conforman el sistema fonológico, la forma cómo estos se organizan para crear unidades mayores y cómo estos son descritos a través de rasgos distintivos. Lo anterior implica que muchos fenómenos de variación están siendo obviados o en el mejor de los casos, tratados indirectamente. En el estudio fonológico no es suficiente los estudios de las unidades y sus 
https://doi.org/10.46744/bapl.201801.006

rasgos distintivos; por ello, es imprescindible conocer la forma en que las unidades se relacionan y cómo el contexto puede generar variación en ellas (Arias, 2007).

Un constituyente importante para el análisis de los segmentos es la sílaba, Núñez et al. (2014) la definen así: «La sílaba se puede definir, pues, como un conjunto de segmentos agrupados en torno a un núcleo (la vocal)» (p. 195). En efecto, la posición que ocupe un segmento en la sílaba determinará su rendimiento funcional y los procesos que pueda sufrir. En otras palabras, en la sílaba se pueden encontrar posiciones «privilegiadas» y posiciones «no-privilegiadas», entre las primeras está el arranque de sílaba y en las segundas está la coda silábica. Las posiciones privilegiadas tienden a mantener los contrastes fonológicos, mientras que las posiciones no- privilegiadas tienden a neutralizar los contrastes fonológicos. Por lo tanto, se implica que el comportamiento de los segmentos en posición de arranque y coda es asimétrico, respecto a ello Jímenez y Lloret (2013) nos comentan: «En el ataque, son universalmente preferibles los segmentos que presentan respecto del núcleo mayor distancia de sonicidad y, en la coda, los que presentan menor distancia (Clements, 1990)» (p. 1). A continuación, (1) presentamos la escala de sonoridad de los segmentos:

(1) Vocales $>$ deslizadas $>$ líquidas $>$ nasales $>$ obstruyentes

De lo anterior se puede implicar que las modificaciones que pueden sufrir los segmentos en posición de ataque son procesos de reforzamiento, mientras que en las codas se dan procesos de debilitamiento. Es decir, es preferible que los segmentos en posición de coda estén más cercanos en sonoridad a la vocal, mientras que en el ataque se prefiere a los segmentos que estén más alejados en términos de sonoridad de la vocal. Por otro lado, una diferencia más entre los ataques y las codas es la preferencia universal por la sílaba $\mathrm{CV}$, con ataque, pero sin coda.

A pesar de la importancia que implica estudios descriptivos, y en la medida de lo posible explicativos, de los procesos fonológicos producto de la posición de los segmentos, son muy pocos los que existen por lo menos 
en el castellano, entre los que se ha podido obtener están los estudios de Arias (2007) que es un trabajo del habla popular de la Ciudad de México, Valiente (2012) del habla del Concejo de Casares de Las Hurdes en donde trata de forma indirecta los procesos que sufren los segmentos producto de la posición que ocupan en la sílaba y, por último, Jimenez y Lloret (2013) en donde estudia la variedad del castellano panameño y algunas variedades de España.

De todo lo anterior, se desprende que existen pocos estudios sobre el problema que el presente trabajo pretende explicar; además, cabe agregar que no existe un estudio similar para alguna variedad del castellano de Perú. Ante esta situación, nos formulamos la siguiente pregunta ¿Cuáles son los procesos que afectan a los segmentos oclusivos en posición de coda en la variedad de castellano limeño? En términos de hipótesis, pensamos que los procesos de debilitamiento que afectan a los segmentos oclusivos en posición de coda al interior de palabra en el castellano limeño son los siguientes: elisión, cambio de sonoridad, fricativización y cambio de punto de articulación. En este artículo, buscamos identificar y explicar los procesos de debilitamiento que afectan a los segmentos oclusivos en coda en el castellano limeño. Específicamente, intentamos describir acústicamente los procesos que afectan a los segmentos oclusivos en coda en el castellano limeño.

\section{La fonología generativa (Sound Pattern of English)}

La fonología generativa tiene como presupuesto básico que los segmentos, es decir, /a, e, p, t, s.../ se pueden descomponer en unidades menores, estas unidades son los rasgos, que se pueden definir como comandos neurales que actúan en conjunto para configurar los sonidos del lenguaje. A continuación, se presentan los rasgos que se postulan en el libro Sound Pattern of English, Chomsky y Halle (1968):

- Rasgos de clase mayor

[+/- sonante]: Son sonidos producidos con una configuración en la cavidad oral, de tal forma que posibilita la sonoridad espontánea. 
[+/- vocálico]: se producen con una constricción máxima que no pasa de las que se encuentran en vocales altas y con las cuerdas vocales, de modo que permitan la sonoridad espontánea.

[+/- consonántico]: se producen con una obstrucción importante (al menos tan marcado como en las fricativas) en la región medio sagital del aparato vocálico.

\section{- Rasgos de cavidad}

[+/- coronal]: se producen con la corona de la lengua.

[+/- anterior]: se producen con una obstrucción delante de o en los alveolos.

- $\quad[+/-$ alto]: se producen elevando el cuerpo de la lengua por encima de su posición neutral.

[+/- bajo]: se producen con el cuerpo de la lengua por debajo de su posición neutral.

[+/- posterior]: se producen retrayendo el cuerpo de la lengua respecto a la posición neutra de esta.

[+/- redondeado]: se producen abocinando los labios.

[+/- distribuido]: se producen con una constricción que se extiende a una considerable distancia a lo largo de la dirección de la corriente de aire.

- Aberturas secundarias

$[+/$-nasal $]$

$[+/$-lateral $]$

- Rasgos de modo de articulación

[+/- continuo]: son aquellos que no presentan una obstrucción suficiente en la cavidad oral para detener el flujo de aire.

[+/- tenso]: mayor o menor control y esfuerzo muscular en la articulación

- Rasgos de fuente

[+/- estridente]: en los estridentes aparece una mayor 
cantidad de energía no sonora aperiódica que en los no estridentes.

[+/-sonoro]: presencia o ausencia de vibración en las cuerdas vocales.

Estos rasgos se almacenan en lo que se denomina matrices de rasgos, donde van todos aquellos pertinentes para diferenciar un segmento de otro. En (2) se verá la estructuración a través de rasgos distintivos de una palabra del castellano.

(2)

$$
\left(\begin{array}{l}
\text { +consonante } \\
\text {-sonante } \\
\text {-sonoro } \\
\text {-coronal } \\
\text { +anterior } \\
\ldots
\end{array}\right)\left(\begin{array}{l}
\text { +vocálico } \\
+ \text { sonante } \\
+ \text { posterior } \\
+ \text { bajo } \\
\text {-alto } \\
\cdots
\end{array}\right)\left(\begin{array}{l}
\text { + consonante } \\
+ \text { sonante } \\
+ \text { nasal } \\
+ \text { coronal } \\
+ \text { anterior } \\
\ldots
\end{array}\right)
$$

Por otro lado, la teoría generativa se ha enfocado en explicar y describir a los componentes de la gramática a través de una teoría de representaciones y un sistema computacional (reglas). A continuación, se cita un fragmento de la tesis de Ulloa (2000) que será crucial para entender el sistema de representaciones con el que trabaja la teoría generativa:

La capacidad humana para el lenguaje está diseñada de tal modo que minimiza cantidad de información que debe ser almacenada (=especificada) en el lexicón mental de un hablante; es decir, para el lenguaje, el almacenamiento de la información léxica es apremiante (Kenstowicz 1994: 59-60).

En efecto, bajo la teoría generativa en la gramática hay dos tipos de representaciones: 
- Representación subyacente: contiene solo los rasgos distintivos, no predecibles.

- Representación superficial: contiene tanto rasgos distintivos como no distintivos.

De lo anterior, se implica que la gramática solo almacena aquella información necesaria para distinguir a un segmento de otro, mientras que en la representación superficial deben estar especificados todos los rasgos que la máquina articulatoria necesita para producir un sonido. Entonces, cabe preguntarse bajo qué mecanismos la gramática especifica los rasgos no distintivos en la representación superficial.

Las reglas son los mecanismos que permiten obtener una forma superficial con especificaciones para todos los rasgos, a partir de una representación subyacente, regida por la economía de rasgos; asimismo, estas son el único puente de relación entre las dos representaciones. De esa forma, se puede explicar cómo palabras como /violeta/ salen a la superficie como [vjo.le.ța]. Como se observa, existe un cambio en el segmento /i/de la forma subyacente que deriva a una deslizada [j], ello se explica con la siguiente regla:

(3) $/ \mathrm{i} / \rightarrow \mathrm{j} / \mathrm{l} /$

La regla (3) básicamente indica que la vocal/i/ se convierte en deslizada cuando antecede a la vocal /o/; esta regla se formaliza de la siguiente forma:

Regla a

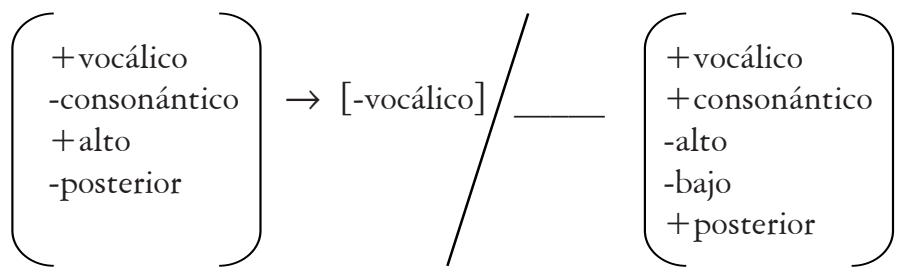


La regla se lee de la siguiente forma: una vocal/i/ cambia el valor de su rasgo [+vocálico] a [- vocálico] cuando le sigue una vocal /o/.

Entonces, en la fonología generativa se busca que las reglas den cuenta de los cambios que existen entre la forma subyacente y la forma superficial; asimismo, la especificación de los contextos de dichos cambios y, por último, se busca que las reglas sean lo más generales posibles para que puedan extrapolarse a fenómenos parecidos en otras lenguas e, incluso, en la lengua misma. Así, al tener reglas generales aplicables a distintos fenómenos la gramática cumple con el requisito de la economía, al no llenarse de reglas para cada fenómeno particular. De todo lo antes dicho, se desprende que el componente fonológico en la teoría generativa lineal está constituido por la representación subyacente, la representación superficial y un conjunto de reglas que explican los procesos que se dan en una lengua determinada.

\section{Metodología}

La presente investigación es de índole descriptivo-explicativa, puesto que se busca describir acústicamente los procesos de debilitamiento que sufren los segmentos oclusivos en posición de coda; asimismo, es explicativa, ya que buscamos explicar por qué se dan los procesos.

En este estudio participaron 5 colaboradores, entre varones y mujeres cuyas edades oscilan entre 18 y 23. Cabe recalcar que dos de los colaboradores son bilingües español-inglés cuya primera lengua fue el español. Cuatro de los colaboradores viven y nacieron en Lima, solo una colaboradora nació en provincia. A continuación, en la tabla 1 se presentan las características más importantes de nuestros colaboradores. Asimismo, a lo largo de este estudio nos referimos a ellos con las etiquetas $\mathrm{H} 1, \mathrm{H} 2, \mathrm{H} 3, \mathrm{H} 4$ y $\mathrm{H} 5$. 
https://doi.org/10.46744/bapl.201801.006

Tabla 1: Características de los colaboradores

\begin{tabular}{|c|c|c|l|}
\hline Colaborador & Género & Edad & Nivel de educación \\
\hline H1 & M & 19 & Superior sin concluir \\
\hline H2 & F & 18 & Superior sin concluir \\
\hline H3 & M & 20 & Superior sin concluir \\
\hline H4 & F & 21 & Superior sin concluir \\
\hline H5 & M & 23 & Superior sin concluir \\
\hline
\end{tabular}

Las entrevistas se llevaron a cabo en un ambiente cómodo. Con la ayuda de una asistente de investigación (una compañera) es que se pudo obtener los datos en su forma más natural. Asimismo, se les explicó a los colaboradores que el objetivo del estudio era estudiar una clase de sonidos de su lengua y que lo que se buscaba era que ellos hablasen de la forma más natural posible, como si estuviesen en una charla de lo más cotidiana con sus amigos. Para lograr la mayor espontaneidad de los datos antes de cada grabación se hablaba con los colaboradores de cualquier tema para entrar en confianza y así obtener datos reales. Además, se les explicó a los colaboradores que la información socio- lingüística que dieran se mantendría anónima y que podrían detener su participación en la investigación cuando lo desearan. También, cabe indicar que las entrevistas se llevaron a cabo en un solo día, en el cual se citó a los colaboradores en distintas horas y que el tiempo por hablante no superó las dos horas.

Por otro lado, nuestro corpus constaba de 50 palabras con los contextos deseados para que los colaboradores puedan elicitar los datos se les entregó unas hojas con el corpus para que puedan leerlo; asimismo, se les dijo que en todo momento usasen una velocidad de habla normal. La palabra objetivo se encontraba dentro de la frase:

(3) $[$ Ahora digo $[\mathrm{X}]$, papá. Yo digo $[\mathrm{X}]$ otra vez, papá $]$

El entrevistado entonces repetía la frase (4) dos veces por palabra objetivo. Los resultados que se presentan, en su mayoría, se basan en la 
primera ocurrencia de la palabra objetivo dentro de la segunda repetición de la frase.

Las grabaciones se realizaron con una grabadora TASCAM. Esta nos permitió grabar audios en formato no comprimido WAV. Asimismo, durante la grabación la grabadora estuvo conectada a un micrófono de tipo diadema que se pone en la oreja como soporte y tiene un pequeño micrófono que va directo a la boca. También, el micrófono se mantuvo a una distancia aproximada entre 4 o $5 \mathrm{~cm}$ de la boca del colaborador.

Por último, el presente estudio utilizó el programa Praat (Boersma y Weenink 2008, versiones 5.0.06 - 5.1.05) para realizar los análisis acústicos.

\section{Descripción de los datos}

Antes de empezar, es menester recalcar que por extensiones del presente estudio solo se tomarán en cuenta, de todo el corpus, las entradas más representativas; es decir, aquellas donde hubo fenómenos que el presente trabajo abarca. Asimismo, en los ejemplos que se mostrarán no solo hubo procesos de debilitamiento de las oclusivas en coda, sino también de debilitamiento en posición intervocálica, incluso elisión de segmentos en ciertas posiciones, estas variaciones han sido tomadas en cuenta en la transcripción para que el estudio sea lo más objetivo posible; sin embargo, como los procesos mencionados antes no competen al tema de este trabajo no serán explicados en el análisis. Por último, se hará una breve descripción acústica de los segmentos oclusivos. Los segmentos oclusivos pertenecen al grupo de los sonidos obstruyentes, puesto que presentan una obstrucción en la cavidad oral. En el caso de las oclusivas la obstrucción es total, es decir, hay un momento en que el aire se ve atrapado en la cavidad oral, se conoce como oclusión y acústicamente se caracteriza por ser una fase sin energía acústica y visualmente como un espacio blanco. Posteriormente, se da la fase de explosión que indica el momento en el que los articuladores se separan y dejan fluir el aire abruptamente a través de la cavidad. Finalmente, el intervalo de tiempo entre la explosión y las vibraciones de las cuerdas 
https://doi.org/10.46744/bapl.201801.006

vocales del segmento que sigue a la oclusiva se conoce como VOT (Voice Onset Time).

\subsection{Oclusiva velar sorda [k] en coda al interior de palabra}

En los siguientes ejemplos se podrán identificar distintas variaciones que afectan a la consonante oclusiva velar sorda $[\mathrm{k}]$ en posición de coda.

\section{Cuadro 1}

\section{H1:/abstrakto/ 'abstracto'}

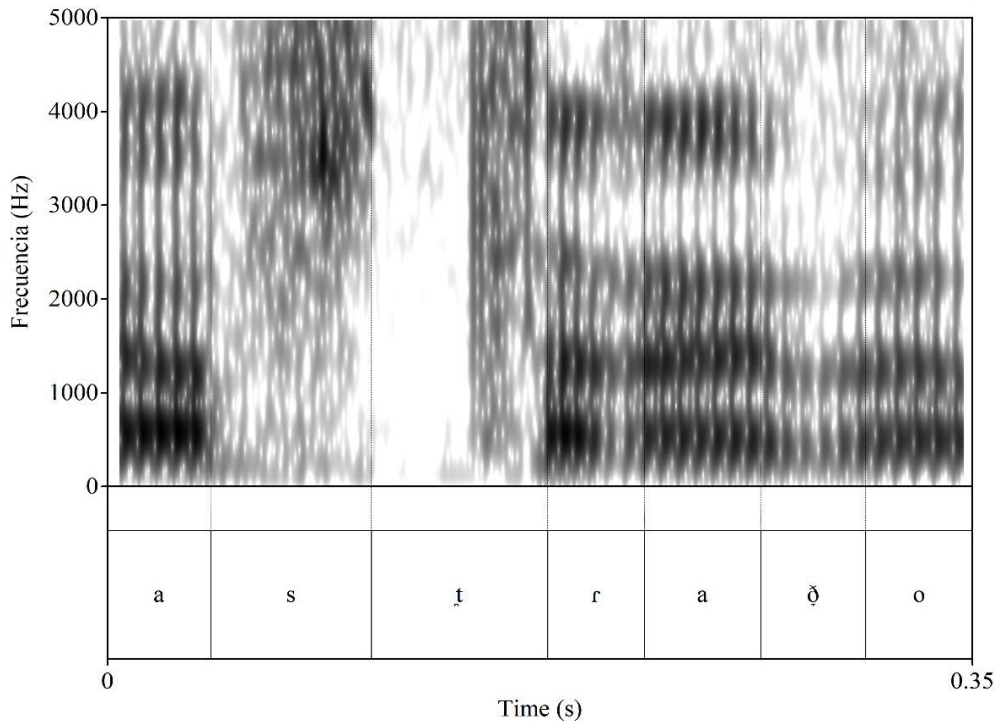

Ignacio (2014): «En buena parte, la evolución fonológica consiste en la reducción de gestos articulatorios (incluyendo la asimilación como un tipo de reducción). El punto último en un proceso de reducción de un segmento es su elisión completa» (p. 103). Como la cita señala, la elisión es un proceso que se enmarca en los procesos de debilitamiento y, en efecto, el cuadro 1 muestra que la oclusiva velar sorda de la segunda sílaba no tiene una correspondencia fonética, puesto que, al observar el espectrograma, la estructura formántica de la vocal /a/ no desaparece; es decir, no hay 
evidencia de una oclusiva y las fases que esta implica (oclusión, explosión y VOT). Asimismo, se descartó la posibilidad de que el segmento en discusión $/ \mathrm{k} /$ se haya espirantizado, dado que la duración del segmento que le sigue a la vocal es de $0,04 \mathrm{~s}$, lo cual imposibilita que haya dos aproximantes juntas. Por último, como es evidente, el segmento/t/ de la forma subyacente pasa a ser una aproximante dental [ð̛] en la forma superficial.

\section{Cuadro 2}

\section{H1: /akseso/ 'acceso'}

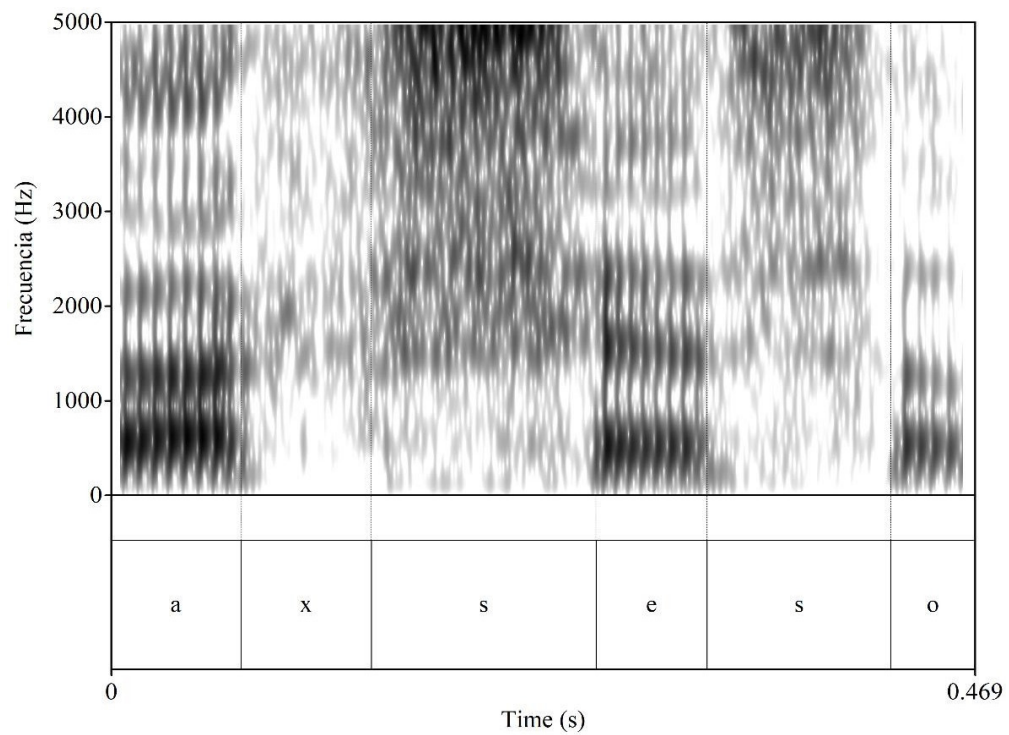

Como se puede observar, el cuadro 2 evidencia un proceso de fricativización correspondiente a la oclusiva $/ \mathrm{k} /$, que en la forma superficial emerge como una fricativa velar $/ \mathrm{x} /$. Naturalmente, los sonidos fricativos se caracterizan por tener una obstrucción parcial, de tal forma que el aire proveniente de los pulmones no encuentra una obstrucción tan marcada como en las oclusivas, como para que el aire sea impedido de salir. Lo anterior implica que al poseer una obstrucción parcial el aire saldrá por un espacio más reducido. Por ello, acústicamente las fricativas se caracterizan por presentar turbulencia, es decir, ruido. Asimismo, cabe indicar que 
las consonantes fricativas sibilantes concentran su energía en frecuencias altas a diferencia de las no sibilantes. Por otro lado, Ulloa (2010) comenta que la energía acústica de las fricativas tiene un patrón aleatorio (es decir, ruido, turbulencia creada por fricción); sin embargo, el modo en el que dicha energía se distribuye en el espectrograma, dependiendo el punto de articulación de estas, no es aleatorio.

Ahora, una de las formas de saber el punto de articulación de un segmento es identificando la frecuencia en la que se ubica el pico más alto de energía de dicho sonido, para este propósito se recurre a los gráficos LPC. Asimismo, es necesario recurrir a una cita de Elías Ulloa en su libro Documentación acústica de la lengua shipibo-conibo (2011):

Conforme el punto de articulación se mueve del frente hacia atrás a lo largo de la cavidad oral, los picos más prominentes de energía acústica se mueven a frecuencias más bajas (Ladefoged y Maddieson, 1996; Johnson, 2003; Ladefoged, 2003, 2005) (p. 88).

Entonces, si la articulación de un sonido se mueve de la parte anterior de la cavidad oral hacia la parte posterior, los picos más prominentes de energía se moverán a frecuencias más bajas. En otras palabras, mientras un sonido sea más anterior, su pico más prominente se ubicará en frecuencias altas. La razón por la cual las frecuencias bajan conforme la obstrucción se produce más atrás en la cavidad oral es debido a que el espacio en frente de la obstrucción se hace más grande y eso genera que el aire vibre a frecuencias más bajas (Ulloa, 2011, p. 109). Así, el pico más prominente de un sonido alveolar se ubicará en frecuencias más altas con respecto a sonidos palatales y velares; por otro lado, el pico más alto de las palatales se ubicará en frecuencias más altas que el de las velares. Cabe recalcar que el criterio antes mencionado no aplica para los sonidos dentales, labiodentales y labiales, puesto que delante de estos no hay un espacio de cavidad considerable por donde el aire pueda vibrar. Es por ello que sus picos más prominentes estarán en frecuencias bajas.

Ulloa brinda un promedio de las frecuencias en el que se ubican los picos más prominentes de los sonidos fricativos del castellano: 
Fricativa alveolar [s]:

Fricativa postalveolar $\left[\int\right]$ :

Fricativa velar $[\mathrm{x}]$ :
$7000-8000 \mathrm{~Hz}$

$4000-5000 \mathrm{~Hz}$

$1000-2000 \mathrm{~Hz}$

Y, efectivamente, elpico más prominente del sonido fricativo velar del cuadro 2 estaba por los 1700-2200 Hz. Sibien los rangos mostrados son relativos, es decir, no son exactos y pueden cambiar en cada hablante, es un hecho que los picos más prominentes no pueden alejarse abismalmente del promedio.

\section{Cuadro 3}

\section{H5: /anekdota/ 'anécdota'}

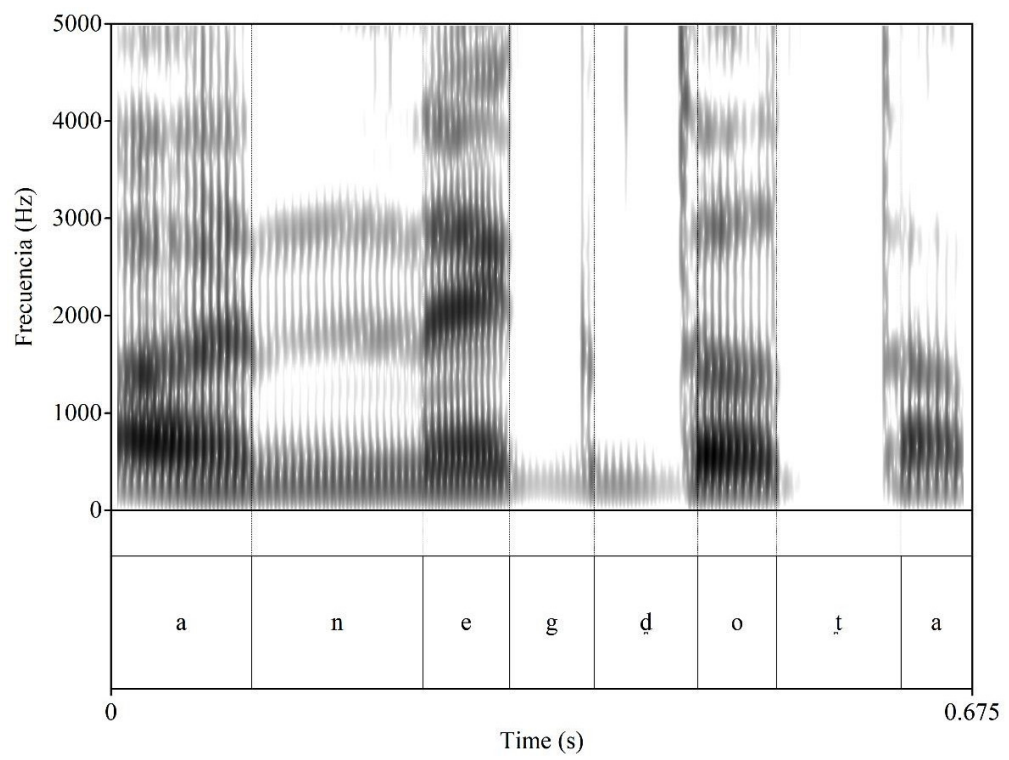

En el ejemplo del cuadro 3 hay un proceso de sonorización: la oclusiva en coda de la segunda sílaba emerge como sonora siendo en la forma subyacente sorda. Como se puede observar, en las oclusivas adyacentes hay energía que está por debajo de los $500 \mathrm{~Hz}$ (barra de voz), esta indica 
https://doi.org/10.46744/bapl.201801.006

la sonoridad de los segmentos. Otro método para saber si un segmento es sonoro o no es a través de la opción pulses $>$ show pulses de praat.

\section{Cuadro 4}

Si se observa el contexto en el que debe estar la oclusiva velar $/ \mathrm{k} /$ de la forma subyacente, solo se puede observar que después de la vocal hay energía distribuida en las altas frecuencias (fricativas); no se puede observar la fase de oclusión ni explosión de las oclusivas, por ello, el cuadro 4 presenta un proceso de elisión de $/ \mathrm{k} /$ al igual que en el cuadro 1. El contraste entre el cuadro 1 y el cuadro 4 es el contexto en el que se da la elisión de /k/; en el primero, a la oclusiva le sigue otra oclusiva que en la forma superficial es un aproximante, mientras que en el segundo a la oclusiva le sigue un segmento fricativo. Asimismo, la elisión de /b/ será explicada en el apartado 4.4 .

\section{H3: /abduksion/ 'abducción'}

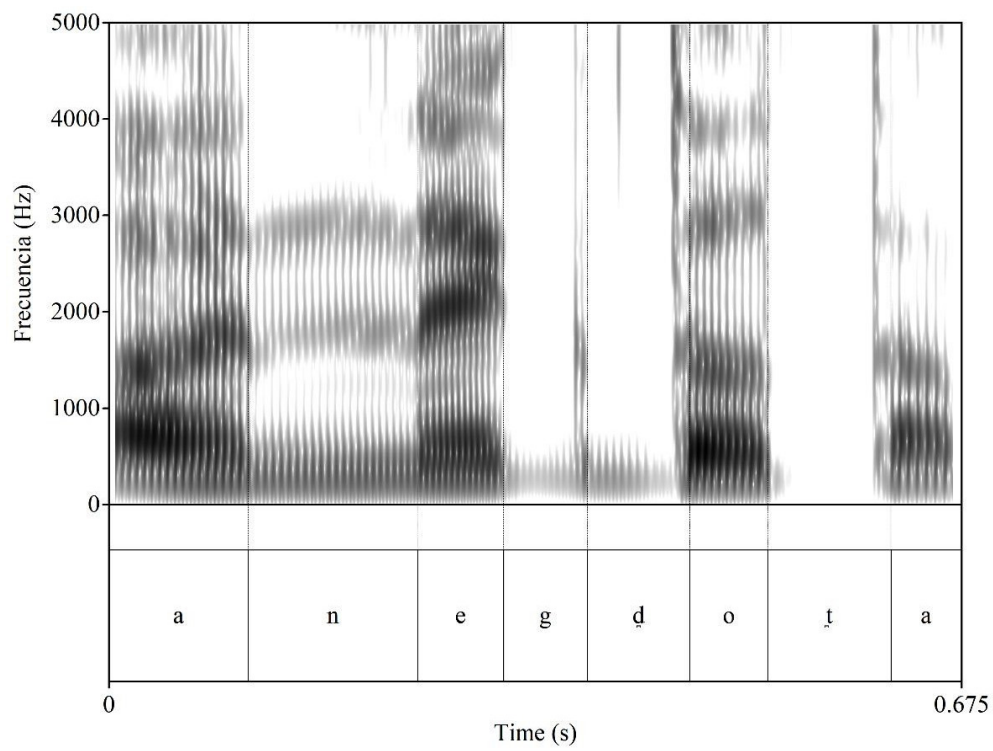

Bol. Acad. peru. leng. 63(63), 2018 / e-ISSN: 2708-2644 


\section{Cuadro 5}

En el ejemplo del cuadro 5, se puede observar que la oclusiva velar de la forma subyacente ha pasado a ser una aproximante velar [ð]], este proceso llamado espirantización es bastante común en las oclusivas en posición intervocálica, pero como el cuadro muestra también se da en la posición de coda. Por otro lado, una característica importante de las aproximantes es que son parecidos a las vocales, en el sentido de que también poseen formantes, aunque como se evidencia en el cuadro los formantes de las aproximantes son menos marcadas que el de las vocales. Además, cabe agregar que este tipo de sonidos son transitorios de o hacia una vocal; por ello, no poseen un periodo donde sus formantes aparezcan estables. Asimismo, es difícil determinar sus fronteras con exactitud. Finalmente, el mismo hablante (H5) espirantizó la oclusiva /k/ en la entrada/teknoloxia/ teniendo como forma superficial a [tę̦.no.lo.xja].

\section{H5: /araknido/ 'arácnido'}

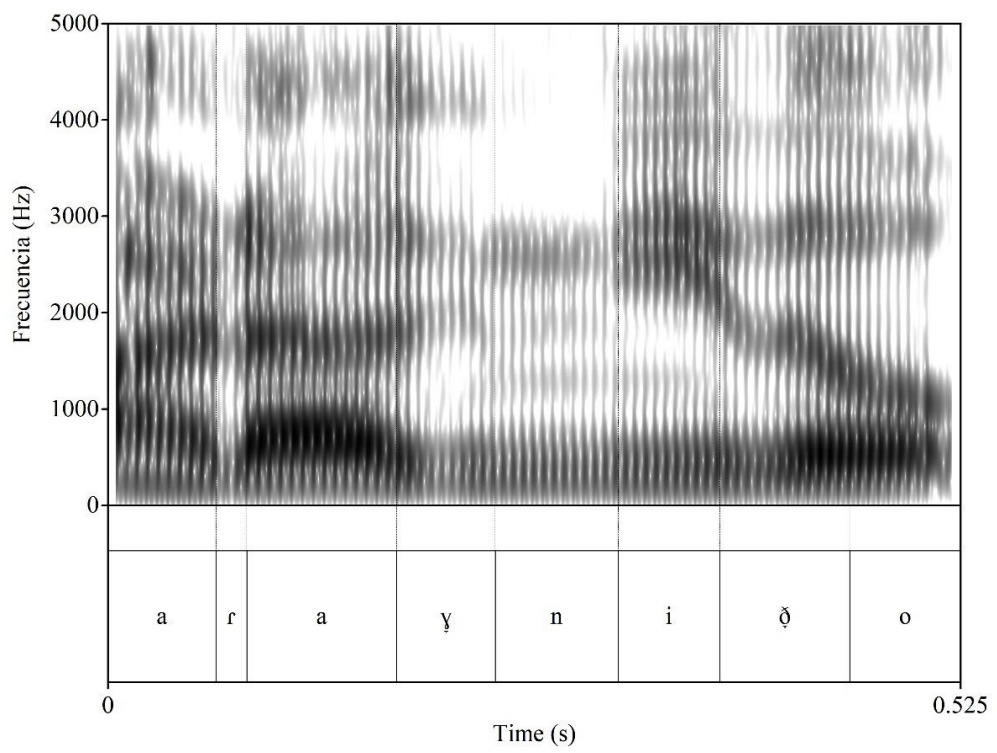


https://doi.org/10.46744/bapl.201801.006

\subsection{Oclusiva velar sonora $[\mathrm{g}]$ en coda al interior de palabra}

A continuación, se presenta un ejemplo que se obtuvo con este sonido:

\section{Cuadro 6}

H4: /cognision/ 'cognición'

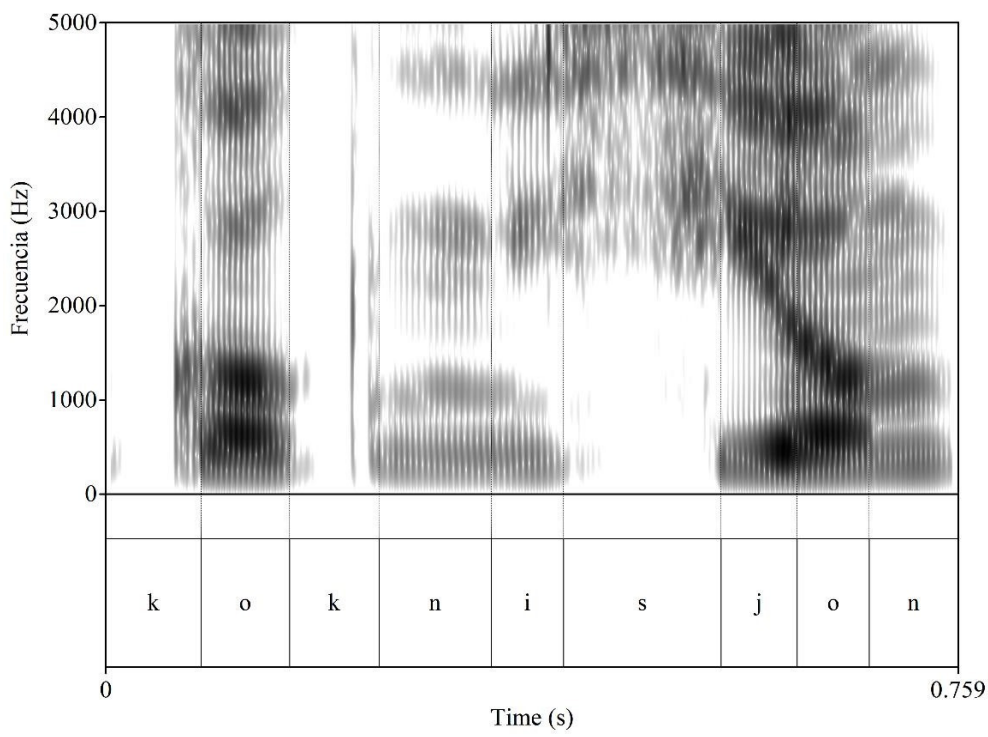

Como se observa, el proceso que se evidencia en el cuadro 5 es la pérdida de sonoridad (ensordecimiento) de $/ \mathrm{g} /$ de la forma subyacente; así, emerge como una oclusiva velar sorda /k/. Acústicamente, los sonidos sordos se caracterizan por la ausencia de la barra de voz; es decir, ausencia de energía por debajo de los $500 \mathrm{~Hz}$. El mismo proceso se encuentra en la entrada/dogma/ y /pigmento/, elicitada por el H4, que emerge como [dokma] y [pikmento], respectivamente.

\subsection{Oclusiva labial sorda [p] en coda al interior de palabra}

A continuación, se presentan ejemplos donde la oclusiva labial $/ \mathrm{p} / \mathrm{de}$ la forma subyacente emerge a la superficie como otro segmento producto de la variación condicionada por la estructura: 


\section{Cuadro 7}

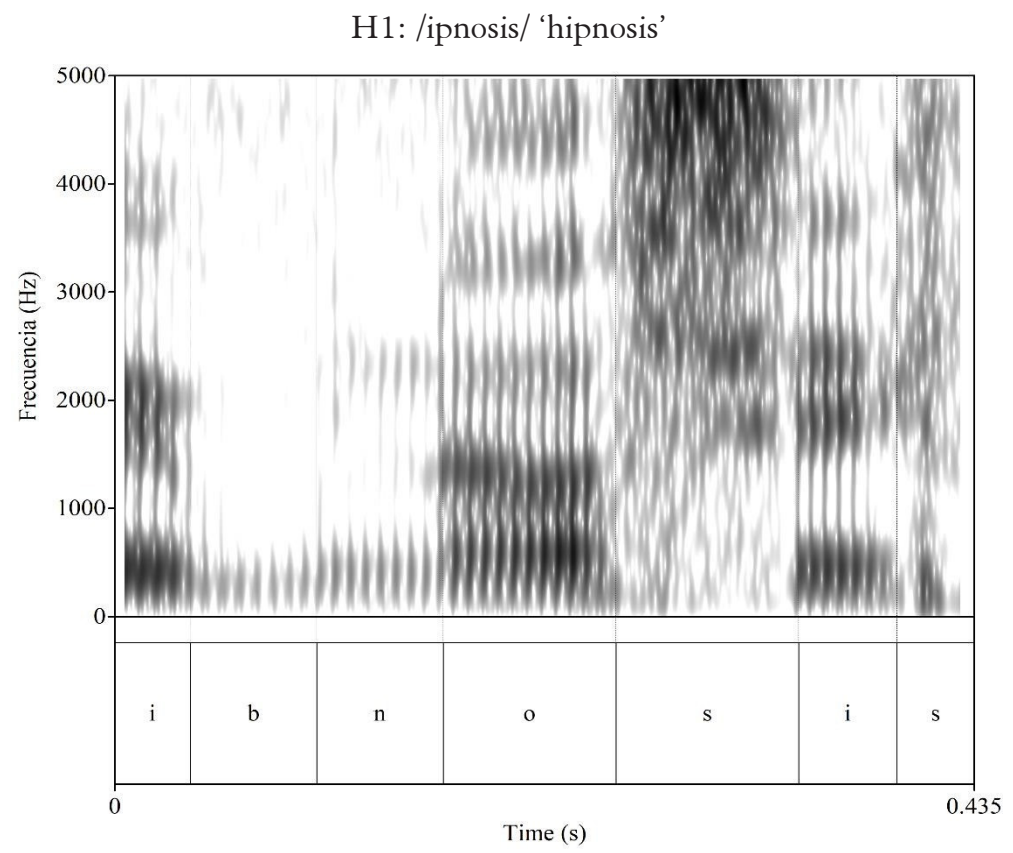

Como se puede observar la oclusiva $/ \mathrm{p} /$ de la forma subyacente emerge como una /b/; es decir, se da un proceso de sonorización y, como se señaló antes, la sonoridad se evidencia a través de la barra de sonoridad.

\section{Cuadro 8}

El cuadro 8 muestra la elisión de la oclusiva /p/ de la segunda sílaba. Es probable que al elidirse el segmento en cuestión haya dejado el tiempo de la oclusión a la oclusiva que le seguía; por ello, en comparación con la oclusiva $/ \mathrm{k} /(0,08 \mathrm{~s})$ del arranque de palabra, la $/ \mathrm{t} /(0,10)$ tiene mayor duración. 
https://doi.org/10.46744/bapl.201801.006

\section{H1: /korupto/ 'corrupto'}

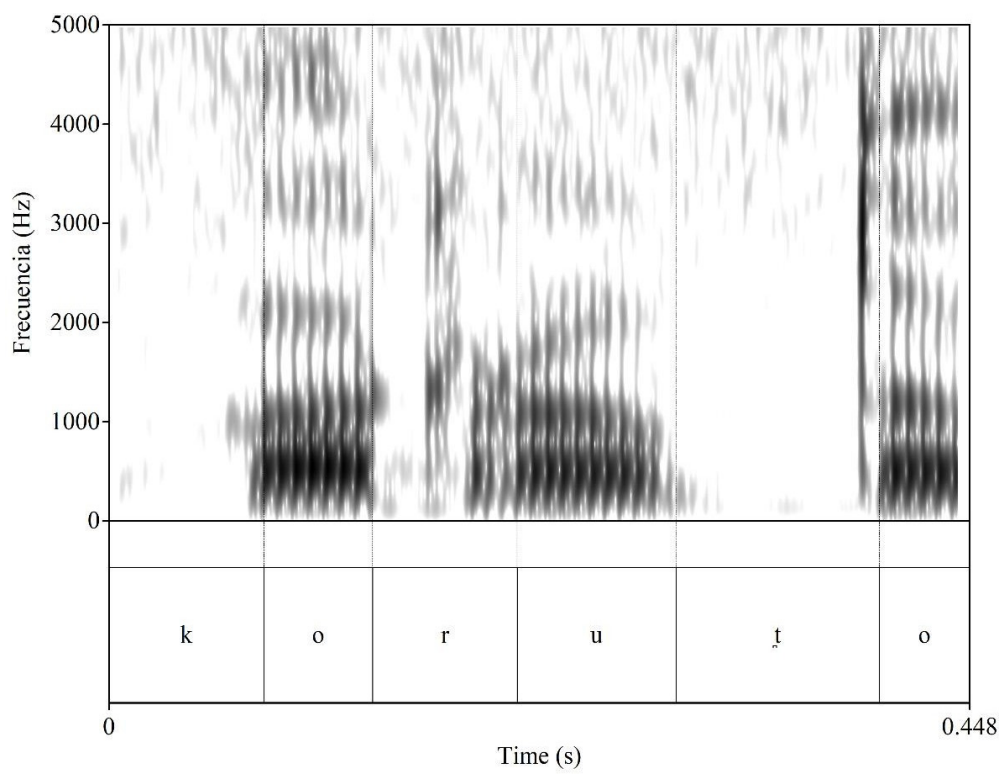

\subsection{Oclusiva labial sonora [b] en coda al interior de palabra}

A continuación, se presentan dos ejemplos donde el segmento /b/ varía en su forma superficial:

\section{Cuadro 9}

En este ejemplo, se puede observar que la oclusiva labial /b/ pasa a ser en la forma superficial una fricativa alveolar sorda. De esta manera, se pueden observar tres procesos: el ensordecimiento, la fricativización y el cambio de punto de articulación. En este caso, el punto de articulación labial de la oclusiva de la forma subyacente emerge como un sonido alveolar fricativo; el método para determinar el punto de articulación de la fricativa ha sido dilucidado en el cuadro 2. 
Óscar Esaul Cueva Sánchez

https://doi.org/10.46744/bapl.201801.006

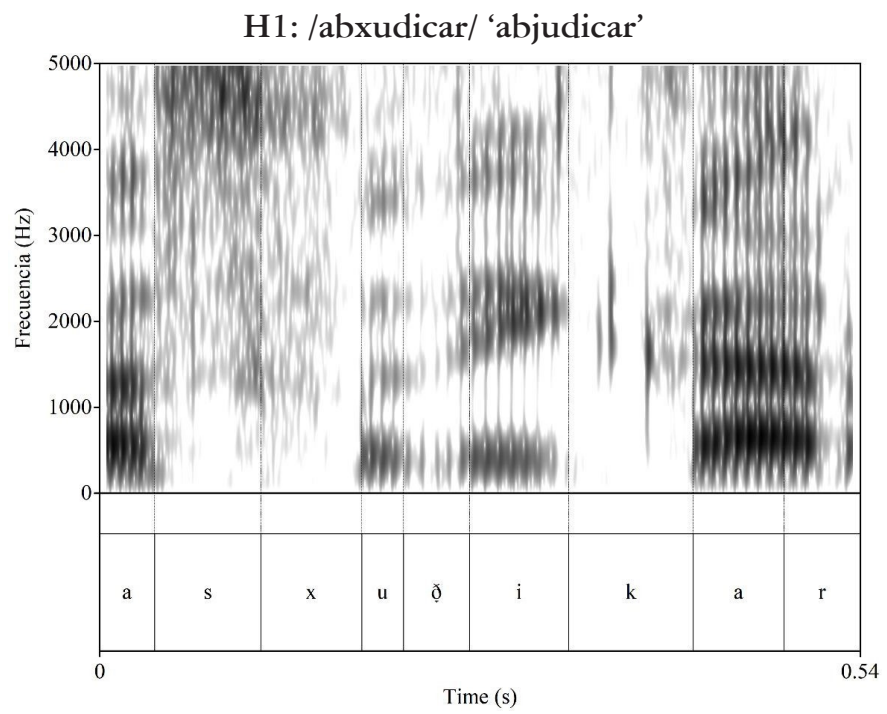

Cuadro 10

H5: /abduksion/ 'abduccion'

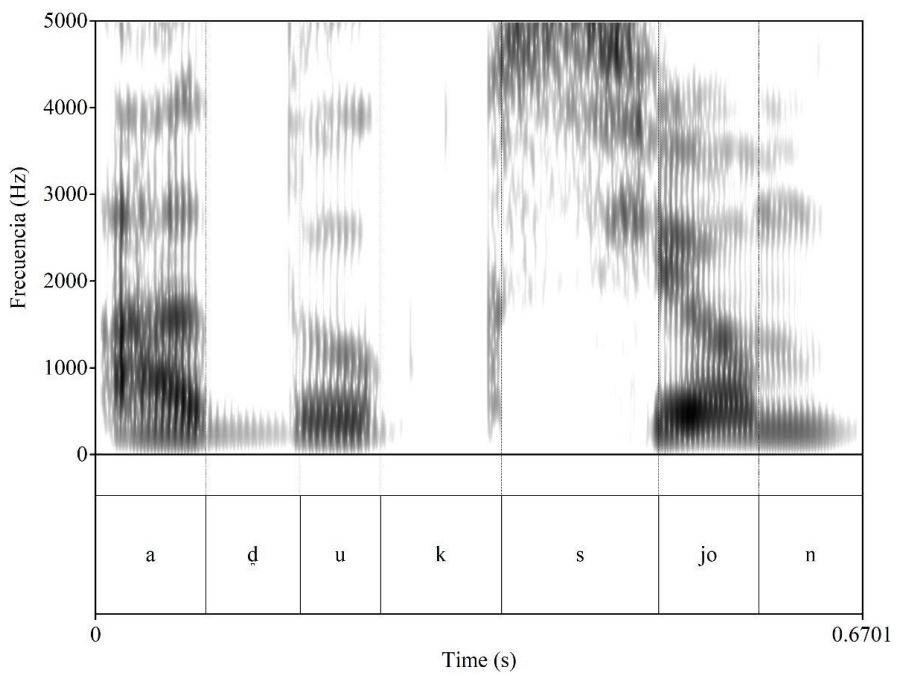


Como se indicó anteriormente, la elisión de la oclusiva labial /b/ de la entrada /abduksion/ será abordada en este apartado. Cabe señalar que la entrada del cuadro 4 fue elicitada por el $\mathrm{H} 3$, mientras que la del cuadro en discusión fue elicitada por el $\mathrm{H} 5$. Se concluye que el proceso presente en el último ejemplo es una elisión, puesto que la duración del segmento adyacente a la vocal es de 0,82 s, lo cual imposibilita la presencia de dos segmentos oclusivos adyacentes, sin recalcar que perceptualmente no hay ningún rastro de la oclusiva labial.

Para finalizar, cabe recalcar que estos son algunos de los procesos encontrados de todo el corpus. Se puede dar el caso en el que la misma entrada elicitada más de una vez por un solo hablante tenga distintos procesos. De lo anterior se implica que los procesos mostrados no son obligatorios en todos los casos, sino que cada hablante puede o no usarlos, ello depende de muchos factores que se escapan del terreno de la lingüística. En lo que resta del trabajo, se formalizarán los procesos de variación mostrados siguiendo los lineamientos de la teoría generativa lineal.

\section{Análisis de las entradas mediante la fonología lineal}

A continuación, se presentan los procesos que se han podido observar en nuestros datos:

a. Fricativización: proceso por el cual un segmento de la forma subyacente pasa a ser fricativo en la forma superficial.

b. Espirantización: proceso mediante el cual un segmento oclusivo de la forma subyacente pasa a ser aproximante.

c. Cambio de sonoridad: proceso mediante el cual un segmento de la forma subyacente, con el rasgo [ $\varphi$ sonoro], pasa a la forma superficial con el rasgo [- $\varphi$ sonoro], donde ' $\varphi$ ' puede tomar el valor positivo $[+]$ o negativo [-].

d. Elisión: proceso mediante el cual un segmento de la forma subyacente no es expresado en la forma superficial.

e. Cambio consonántico: Proceso mediante el cual un segmento, de la forma subyacente, con un punto de articulación (P. A.) 
'X', emerge a la superficie con un P. A. 'Y'.

A continuación, se presentan la forma subyacente y la forma superficial, respectivamente, de los datos obtenido en el capítulo 4, y los procesos que sufre el segmento oclusivo en posición de coda:

\section{Oclusiva velar sorda $[\mathrm{k}]$}
a. /abstrakto/ $\rightarrow$ [as.'tra.ợo] (elisión)
b. /akseso/ $\rightarrow \quad$ [ax.'se.so] (fricativización)
c. /anekdota/ $\rightarrow$ [a.'neg.do.ta] (sonorización)
d. /abduksion/ $\rightarrow$ [a.du.'sjon] (elisión)
e. $/$ araknido/ $\rightarrow \quad$ [a.' rą̦.ni. ָ̦o] (espirantización)

\section{Oclusiva velar sonora $[\mathrm{g}]$}
a. $/$ kognision/ $\rightarrow$
[kok.ni.'sjon] (ensordecimiento)

\section{Oclusiva labial sorda $[\mathrm{p}]$}
a. /ipnosis/ $\rightarrow$
[ib.' no.sis] (sonorización)
b. $/$ korupto/ $\rightarrow$
[ko.ru.to] (elisión)

\section{Oclusiva labial sonora $[\mathrm{b}]$}
a /abxudikar/ $\rightarrow$ [as.xu.ðָi.'kar] (Fricativización, ensordecimiento y cambio de punto de articulación)
b /abduksion/ $\rightarrow$ [a.duk.' sjon] (elisión)

\subsection{Formalización de los procesos}

El presente estudio analiza los procesos de debilitamiento que sufren los segmentos oclusivos en posición de coda, por ello, todas las reglas estarán en dicho contexto. Como anteriormente se indicó, la lengua tiende a la economía, por tanto, las reglas tienen que formularse con la cantidad mínima de rasgos, y estas tienen que ser lo más generales posibles. A 
continuación, se formalizarán las reglas con base en los rasgos explicados en el apartado 2:

\subsubsection{Fricativización}

Para empezar, el rasgo que distingue a sonidos oclusivos de fricativos es el rasgo [+/-continuo], en efecto, los sonidos oclusivos son [-continuos], y los sonidos fricativos son [+continuos]. Por otro lado, el rasgo [+/-sonante] es positivo para vocales, aproximantes, líquidas y nasales, mientras que para obstruyentes (oclusivas, fricativas y africadas) es negativo, dicho rasgo expresará que el tipo de sonido que varía es una obstruyente y no una vocal o una líquida. También, se aclara que la teoría usa un conjunto de símbolos para dar cuenta de los contextos; en este caso, se usará el símbolo \$ que significa límite silábico

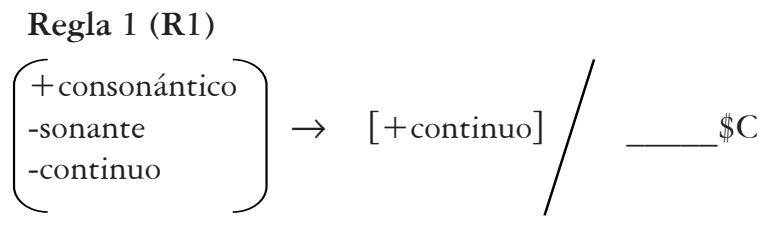

La Regla 1 expresa que un sonido [-sonante] y [-continuo] (oclusiva) pasa a ser [+ continuo] (fricativo) en posición de límite silábico seguido por una consonante (posición coda al interior de palabra).

\subsubsection{Espirantización}

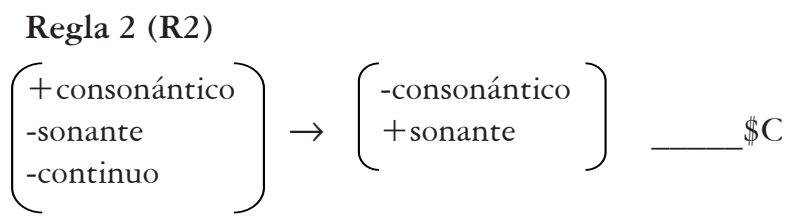

En este proceso, intervienen los rasgos [+/-consonántico] y [+/-sonante], así, una aproximante se caracteriza por ser [-vocálico], [-consonántico $]$ y $[+$ sonante $]$, mientras que un sonido oclusivo se caracteriza por ser [+ consonántico] y [-sonante]. Como se evidencia, los rasgos que intervienen en el proceso son los dos últimos, puesto que el 
rasgo vocálico presenta la misma especificación tanto para aproximantes como para oclusivas.

\subsubsection{Cambio de sonoridad}

En este proceso el único rasgo que varía es [+/-sonoro] y como se señaló antes, la lengua tiende a tener reglas generales. Por ello, se usará variables para representar en una sola regla los procesos de sonorización y ensordecimiento.

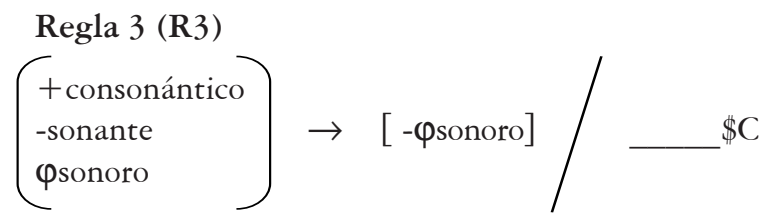

En caso de que $\varphi$ fuese el valor positivo [+], ello indicará que en la forma superficial el valor para [+/-sonoro] será negativo [-], puesto que $[+] *[-]=[-]$. Por otro lado, si $\varphi$ fuese el rasgo [-], ello indicará que en la forma superficial el valor para $[+/$ - sonoro $]$ será $[+]$; ya que [-] $*[-]$ $=[+]$. De ahí que con una sola regla se explica un proceso que puede ir en dos direcciones, es decir, o ensordecer o sonorizar.

\subsubsection{Elisión}

Básicamente este proceso se representa de la siguiente forma:

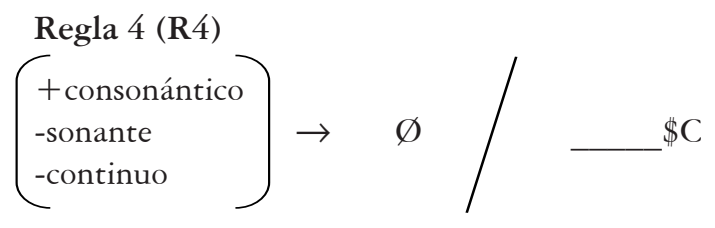

Lo que dice la regla es que un segmento oclusivo en posición de coda silábica seguida por una consonante desaparece/se elimina. Donde $\varnothing$ significa que el segmento no tiene expresión fonética en la superficie. 
https://doi.org/10.46744/bapl.201801.006

\subsubsection{Cambio consonántico}

Se ha encontrado un caso de cambio consonántico en el que un segmento /b/ pasa a ser/s/; es decir, pasa de ser labial a alveolar. Cabe señalar que, de encontrarse casos en los que el cambio de punto de articulación sea más rico; es decir, haya cambios hacia el articulador labial, alveolar o velar, la generalización se vería afectada; puesto que las variaciones motivadas por la posición y no por el contexto tienden a ser más particulares.

\section{Regla 5 (R5)}

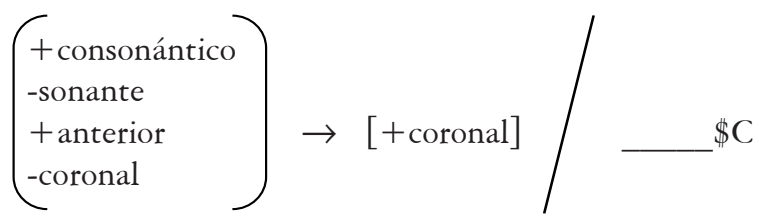

Los sonidos labiales son [+anterior] y [-coronal], mientras que los segmentos alveolares son [+anterior] y [+coronal], por ello, el único rasgo que varía de la forma subyacente a la superficial es el rasgo [+/-coronal $]$.

\subsection{Aplicación de las reglas a los datos}

A continuación, se presenta la derivación de las formas subyacentes: de nuestros datos más representativos a las formas superficiales, y para esto se usarán las reglas ya presentadas (R1, R2, R3, R4 y R5). Es necesario recalcar que nuestras reglas solo sirven para explicar los procesos de variación que sufren los segmentos oclusivos en posición de coda; por ello, cualquier otro fenómeno de variación que no esté enmarcado dentro del tema de estudio será ignorado.

\section{Tabla 1}

\begin{tabular}{|c|l|}
\hline Forma subyacente & /abstrakto/ \\
\hline R4 & [as. 'tra. țr] \\
\hline Forma superficial & [as. 'tra.ðָo] \\
\hline
\end{tabular}


https://doi.org/10.46744/bapl.201801.006

Tabla 2

\begin{tabular}{|c|c|}
\hline Forma subyacente & /akseso/ \\
\hline R1 & {$[$ ax.'se.so] } \\
\hline Forma superficial & {$[$ ax.'se.so] } \\
\hline
\end{tabular}

Tabla 3

\begin{tabular}{|c|c|}
\hline Forma subyacente & /araknido/ \\
\hline $\mathrm{R} 2$ & [a.' ray.ni.do] \\
\hline Forma superficial & [a.' 'ray.ni.ðָo] \\
\hline
\end{tabular}

Tabla 4

\begin{tabular}{|c|c|}
\hline Forma subyacente & /ipnosis/ \\
\hline R3 & {$[$ ib. 'no.sis] } \\
\hline Forma superficial & {$[$ ib. 'no.sis] } \\
\hline
\end{tabular}

Tabla 5

\begin{tabular}{|c|c|}
\hline Forma subyacente & /abxudikar/ \\
\hline R1 & {$[$ a $\beta . x u . d i . k a r]$} \\
\hline R5 & {$[$ az.xu.di.kar] } \\
\hline R3 & {$[$ as.xu.di.kar] } \\
\hline Forma superficial & {$\left[\right.$ as.xu.ð̄i. ${ }^{\prime}$ kar $]$} \\
\hline
\end{tabular}


La aplicación de las reglas a nuestros datos deja abierta una gran discusión: ¿las reglas tienen o no ordenamiento? Por la extensión del trabajo no se ahondará en la cuestión, pero se puede comentar que en el marco de la teoría generativa este tema es bastante polémico, puesto que, si el lector es astuto y «juega» un poco con el ordenamiento de las reglas, puede darse cuenta de que en algunos casos mantener un orden en la aplicación de las reglas es necesario, sino se pueden generar estructuras anómalas para la lengua; sin embargo, en otros casos el orden de las reglas no es necesario, puesto que invirtiendo el orden propuesto la forma superficial siempre será la misma.

Para concluir con este capítulo, se quiere recalcar que las reglas propuestas párrafos atrás no son de carácter obligatorio; es decir, si hay un segmento en posición de coda no significa que obligatoriamente tiene que espirantizarse o elidirse. Asimismo, se puede indicar que los procesos que afecten a las oclusivas en posición de coda están en una especie de variación libre. Es decir, este proceso no depende netamente del plano lingüístico, sino que puede depender también de variables sociales o del tipo de habla que se use en determinado contexto. Por extensiones del estudio, dichas variables no podrán ser analizadas con rigurosidad, por ende, aquello queda para investigaciones posteriores.

\section{Conclusiones}

En primer lugar, se puede decir que el castellano no escapa de la tendencia universal de las lenguas por tener sílabas $\mathrm{CV}$, ello quedó demostrado porque uno de los procesos que afectaban a los segmentos oclusivos en posición coda fue la elisión; asimismo, se comprobó nuestra tesis principal, es decir, que en los segmentos en la posición de coda tienden a estar más cercanos en niveles de sonoridad a los núcleos o vocales, de tal forma que nuestra jerarquía de sonoridad quedaría como en (5):

(5) Jerarquía de sonoridad (de menos perceptible a más perceptible)

oclusivas sordas $>$ oclusivas sonoras $>$ africadas sordas $>$ africadas sonoras 
$>$ fricativas sordas $>$ fricativas sonoras $>$ nasales $>$ líquidas $>$ aproximantes $>$ vocales

Solo que agregaríamos a nuestra jerarquía la elisión como el proceso último que culmina nuestra jerarquía en pro de la sílaba CV. También, se puede concluir que el castellano limeño no es ajeno a los procesos que afectan a los segmentos oclusivos en coda en las distintas variedades de castellano estudiadas. El castellano limeño presenta un gran parecido con el castellano de la Ciudad de México, puesto que presentaban los mismos procesos a excepción de la vocalización presente solo en el castellano de Ciudad de México.

Para finalizar, nuestra hipótesis fue comprobada, ya que todos los procesos que postulamos se evidenciaron acústicamente, incluso nuestra hipótesis resultó rebatida, puesto que se encontró un proceso más: la espirantización. Por último, queremos recalcar que estos procesos están en variación libre, son como un haz de procesos que la gramática escoge 0 no dependiendo del contexto, de variables sociales, entre otros. El hecho de que un proceso se dé o no escapa de las explicaciones lingüísticas para redirigirse a los planos sociolingüísticos, en este radica la respuesta al por qué nuestra colaboradora (H2) nunca usó procesos de debilitamiento. En términos muy simples y poco rigurosos, me arriesgo a afirmar que H2 no elicitó ningún proceso, debido a que estudia artes escénicas y usa constantemente un habla cuidada, marcada o más formal que los otros colaboradores. 
https://doi.org/10.46744/bapl.201801.006

\section{BIBLIOGRAFÍA}

ARIAS, B. (2007). Estudio sobre la realización de algunas consonantes en coda en el habla popular de la Ciudad de México: hacia una interpretación causal de los hechos. Boletín de filología, Tomo XLXX, 11-35.

CHOMSKY, N. \& HALLE, M. (1968). The Sound pattern of English (José Millán, trad.). Madrid: Fundamentos.

IGNACIO, J. (2014). Los sonidos del español. New York: Cambridge University Press.

JÍMENEZ, J. \& LLORET. (2013). Sonicidad y tipología de los márgenes silábicos en español. España: Universitat de Valencia; Universidad de Barcelona.

JIMENEZ, J. (2018). Los sonidos de la lengua A rabela: un bosquejo fonológico. Lima: Universidad Nacional Mayor de San Marcos.

ULLOA, E. (2011). Una documentación acústica de la lengua shipibo-conibo (Pano). Lima: Fondo Editorial de la Pontificia Universidad Católica del Perú.

VALIENTE, A. (2012). Aplicación de la teoría de la optimalidad al consonantismo del habla de Concejo de Casares de las Hurdes. Anuario de estudios filológicos, Vol XXXV, 235- 253. 